\title{
Editorial
}

\section{Precision Medicine for Visceral Cancer: Does the Future Lie in Molecular Stratification?}

\author{
Hauke Lang ${ }^{a}$ Florian Lordick ${ }^{b}$ \\ ${ }^{a}$ Department of General, Visceral, and Transplantation Surgery, University Hospital Mainz, Mainz, Germany; \\ ${ }^{b}$ Department of Oncology, Gastroenterology, Hepatology, Pulmonology, and Infectious Diseases, Leipzig University \\ Medical Center, Leipzig, Germany
}

Visceral cancers represent a highly heterogeneous group of malignancies with regard to pathogenesis, phenotype, clinical presentation, as well as their molecular profile. Currently, multidisciplinary treatment is the standard of care for most visceral tumors. This approach relies on a close cooperation between all disciplines involved in cancer management, ranging from diagnosis over treatment to long-term follow-up.

Surgical resection is still the central component of treatment, even more as cure without surgery occurs rarely. Improved surgical strategies, based on technical refinements and better knowledge of tumor growth patterns, have led to the adoption of more oncologically adequate resections as well as more functionally oriented reconstructions. This results not only in prolongation of survival but also in better quality of life. In addition, subspecialization has evolved and contributes to a higher quality of care. Today, surgeons with a broad knowledge in surgical oncology typically focus on the treatment of malignancies of one or at most a few organs. This is an important step towards precision medicine for visceral cancer surgery. Similarly, and concomitant to the advances in surgery, there has been considerable progress in all other disciplines involved in cancer treatment. We have seen important advances in the diagnostic workup, including imaging and endoscopic techniques, perioperative management, radiotherapy and other locoregional interventions, and - last but not least - in the introduction of more effective chemotherapies and targeted therapies. Results from multicenter clinical trials investigating the effects of multimodal treatment have changed paradigms and the landscape of care. All these achievements led to significant improvements in the prognosis of patients suffering from visceral cancer.

Nevertheless, the results of current treatments are not yet satisfactory, as many patients still present with early tumor relapse. To better identify patients who may benefit from specific therapies, prognostic scores have been developed for qualified treatment stratification [1]. Prognostic scoring based on clinicopathological characteristics has been shown to be helpful, e.g., in predicting outcome after surgery in general, but currently employed scoring systems are inconsistent in accurately determining prognosis in an individual patient. In other words, patients who seem to have "the same disease and the same extent of disease" often show extremely different outcomes despite receiving similar or even the same treatment. This is not at all surprising, as classification on the basis of traditional items such as T, N, and M categories often results in very heterogeneous groups biologically. As such, subgroups that would be in need of a specific treatment are likely to remain undetected, as are subgroups who may receive ineffective overtreatment [2]. This is particularly true for visceral cancer with its extraordinary heterogeneity with respect to genetic and molecular profiles [3].

In recent years, the analysis of cancer-related genes and their mutations using next-generation sequencing and other technologies has extended considerably the spectrum of predictors for prognosis in many visceral cancers. For instance, in colorectal liver metastases, enhanced molecular profiling, mainly aiming at mutations 
of TP53, the RAS/RAF pathways, or the SMAD family, proved to be helpful to better stratify patients eligible for surgery $[4,5]$.

It is very likely that a better knowledge of the molecular pattern will promote the identification of factors influencing outcome after surgery in many visceral cancers. These data may help to understand why patients with "similar disease and treatment" can show variable clinical courses.

The deployment of targeted therapies is the greatest immediate benefit of molecular sequencing. The identification of microsatellite instability, to give a recent example, paves the way for offering much better treatment with PD-1 inhibitors (programmed cell death protein 1) compared to conventional chemotherapy. Unfortunately, European and national drug approval agencies sometimes lack considerably behind the progresses seen in the precision medicine era. In addition, the knowledge of the molecular tumor profile may also have an influence on the general treatment strategy. Contemporary examples are the choice of upfront versus delayed surgery after neoadjuvant therapy, and even the choice of specific surgical techniques and the extent of surgery, e.g., anatomical versus nonanatomical resection, depend on the mutational status of colorectal liver metastases, for example [5].

The development and implementation of precision medicine is in its beginning, certainly work in progress, and still in very different stages in the distinct tumor entities. While molecular profiling of colorectal liver metastases has already some specific impact on the choice of the treatment strategy, therapy of hepatocellular carcinoma is still based on classical parameters such as tumor number and size as well as tumor response to locoregional therapy (not even to chemotherapy). In contrast, recent results of immune therapy in irresectable hepatocellular carcinoma are promising, and treatment of intrahepatic cholangiocarcinoma has even become a hallmark of modern precision medicine approaches - at least in the palliative setting - based on better detection of targetable molecular alterations. Current debates include the questioning of perioperative chemotherapy for microsatellite instability-high gastric adenocarcinoma or the best approach to HER2-positive advanced colon and biliary tract cancers [6].

The present special Issue aims to provide a comprehensive overview on the status quo of personalized treatment for patients with visceral cancers. It covers the pathological background as well as treatment options of visceral cancers - ranging from systemic or locoregional palliative treatment to potentially curative surgical approaches. Special emphasis is placed on future directions in their interdisciplinary management with focus on molecular profiling and potential use of targeted therapy and immunotherapy.

\section{Conflict of Interest Statement}

Hauke Lang and Florian Lordick have no conflicts of interest.

\section{Funding Sources}

There have been no other funding sources.

\section{Author Contributions}

Both authors contributed equally to the editorial.

\section{References}

1 Fong Y, Fortner J, Sun RL, Brennan MF, Blumgart LH. Clinical score for predicting recurrence after hepatic resection for metastatic colorectal cancer: analysis of 1001 consecutive cases. Ann Surg. 1999 Sep;230(3):309-18.

2 Moris D, Pawlik TM. Personalized treatment in patients with colorectal liver metastases. J Surg Res. 2017 Aug;216:26-9.
3 Barbon C, Margonis GA, Andreatos N, Rezaee N, Sasaki K, Buettner S, et al. Colorectal liver metastases: does the future of precision lie in genetic testing? J Gastrointest Surg. 2018 Jul;22(7):1286-96.

4 Lang H, Baumgart J, Heinrich S, Tripke V, Passalaqua M, Maderer A, et al. Extended molecular profiling improves stratification and prediction of survival after resection of colorectal liver metastases. Ann Surg. 2019 Nov;270(5):799-805.
5 Vauthey JN, Kawaguchi Y. Innovation and future perspectives in the treatment of colorectal liver metastases. J Gastrointest Surg. 2020 Feb;24(2):492-6.

6 Lordick F. Chemotherapy for resectable microsatellite instability-high gastric cancer? Lancet Oncol. 2020 Feb;21(2):203. 